\title{
Prospects for Non-Rare Earth Permanent Magnets for Traction Motors and Generators
}

\author{
M.J. KRAMER, ${ }^{1,2,4}$ R.W. McCALLUM, ${ }^{1,2}$ I.A. ANDERSON, ${ }^{1,2}$ \\ and S. CONSTANTINIDES ${ }^{3}$ \\ 1.-Department of Materials Science and Engineering, Iowa State University, Ames, IA 50011, \\ USA. 2.-Ames Laboratory, U.S. Department of Energy, Iowa State University, ISU Campus, \\ Ames, IA 50011, USA. 3.-Arnold Magnetic Technologies Corporation, Rochester, NY 14625, USA. \\ 4.-e-mail: mjkramer@ameslab.gov
}

With the advent of high-flux density permanent magnets based on rare earth elements such as neodymium (Nd) in the 1980s, permanent magnet-based electric machines had a clear performance and cost advantage over induction machines when weight and size were factors such as in hybrid electric vehicles and wind turbines. However, the advantages of the permanent magnet-based electric machines may be overshadowed by supply constraints and high prices of their key constituents, rare earth elements, which have seen nearly a 10 -fold increase in price in the last 5 years and the imposition of export limits by the major producing country, China, since 2010 . We outline the challenges, prospects, and pitfalls for several potential alloys that could replace Nd-based permanent magnets with more abundant and less strategically important elements.

\section{INTRODUCTION}

Transportation accounts for $28 \%$ of energy consumption in the United States ${ }^{1}$ of which $93 \%$ comes from petroleum. Options for replacing fossil fuels for transportation are limited. While biofuels and hydrogen can replace fossil fuels for use in internal combustion engines, electric motors in combination with an internal combustion engine or all-electric vehicles are seen as the primary technologies capable of reducing dependence on petroleum for the transportation sector. Since the advent of high-flux density permanent magnets based on rare earth elements such as neodymium $(\mathrm{Nd})$ or samarium $(\mathrm{Sm})$, permanent magnet-type electric machines* are preferred over induction machines when weight and size are factors, such as in hybrid electric vehicles. ${ }^{2}$ For electric generation, wind is one of the more cost-effective renewable energy sources. Permanent magnet-based-systems have several advantages over induction machines that are heavier, noisier and require more maintenance

\footnotetext{
*Electric machines, a classification that encompasses both motors and generators, are ubiquitous in the modern industrial economy spanning power output from a few milliwatts to megawatts.
}

due to their high-ratio gear boxes. ${ }^{3,4}$ However, the advantages of the permanent magnet-based electric machines maybe overshadowed by the current limited availability and price of their key constituent, rare earth elements. ${ }^{5-9}$ We will present an overview of the past, present, and future prospects for magnet alloys used in permanent magnet-based electric machines in light of current market pressures on rare earth elements and prospects for replacing alloys based on rare earth elements with more abundant and less strategically critical elements.

Permanent magnets are arguably one of the earliest functional materials. Lodestones, comprised mainly of magnetite, were known in ancient times. ${ }^{10}$ The magnetic properties of carbon steel were first reported in the 1600 s by Sir William Gilbert. With the discovery of electromagnetism in the early 19th century by André-Marie Ampère and the formalism of Maxwell's equations, the stage was set for the linking of mechanical work to electromagnetism so that by the end of the $1800 \mathrm{~s}$, simple electric motors and generators were in production. In fact, all-electric and hybrid automobiles were commercially available more than 100 years ago with the first hybrid built in 1900 by Ferdinand Porsche. The drawback to electric cars was their 


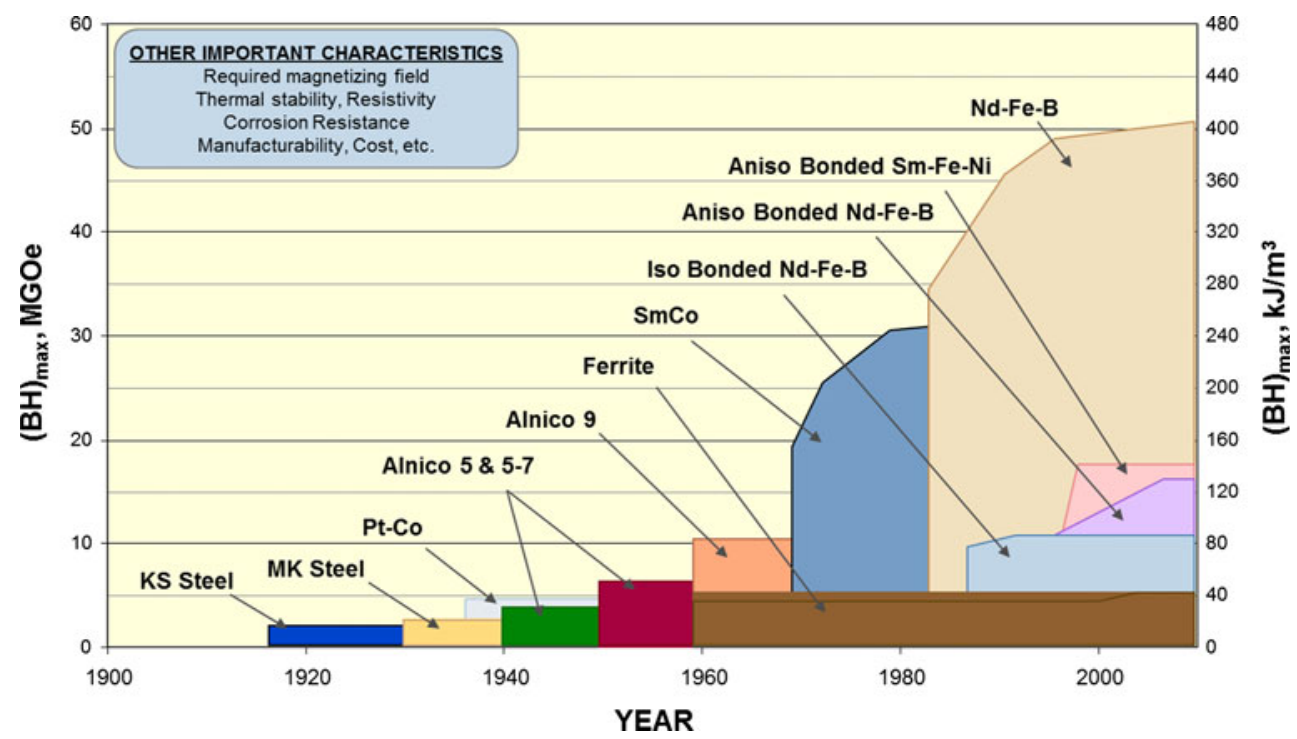

Fig. 1. Historical trend of the improvement in the maximum energy product for commercially produced permanent magnets.

short range due to low battery capacity and low efficiency of the electric motors. There have been significant advances in both these areas with the most efficient motors for vehicle applications being permanent magnet motors made possible by highperformance permanent magnets. A permanent magnet must have a large magnetic induction $(B)$ and the ability to maintain $\mathrm{B}$ in a fixed direction relative to the body of the magnet in the presence on an opposing magnetic field $(H) . B$ as a function of $H$ is given by $B(H)=M(H)+H$, where $M(H)$ is the magnetization of the material as a function of $H$. The energy product of a magnet is the product of $B(H)$ and $H$ The largest negative value of this product when $B(H)$ and $H$ are opposed is $(B H)_{\max }$, which is used as a figure of merit. It is a measure of the maximum amount of work that can be performed by a permanent magnet in units of megagauss oersteds (MGOe, or $\mathrm{kJ} / \mathrm{m}^{3}$ in SI units). The ability of a magnet to maintain its magnetization in the presence of an opposing magnetic field is referred to as its intrinsic coercivity. It is characterized by the value of $H$ required to reduce $M(H)$ to zero, $H_{\mathrm{cJ}}$ is referred to as the intrinsic coercivity.

Through the first half of the $1900 \mathrm{~s}$, most permanent magnets were Fe-based alloys. The first significant improvement in the maximum energy product was achieved in columnar grown alnico (Fig. 1). The improvement came about from the grain alignment of the cubic phases and the application of a magnetic field during cooling through the spinodal decomposition into the nominally $\mathrm{FeCo}$ body-centered cubic (bcc) and the primitive cubic $\beta$-AlNi phases. ${ }^{11}$ This produces what is essentially an aligned array of nanoscaled compass needles in a nonmagnetic matrix. These Fe-based alloys have sufficient intrinsic magnetization from the $\mathrm{Fe}$ and Co atoms, which results in measurable residual induction $\left(B_{\mathrm{r}}\right)$, whereas the shape of the needles makes it favorable for the magnetization to point along the axis of the needle, resulting in shape anisotropy. Improving the resistance of an alloy to demagnetizing fields, i.e., $H_{\mathrm{cJ}}$, was paramount to improving energy product in Fe-based alloys. While $\mathrm{PtCo}$ and ferrites were significant discoveries in the mid-20th century, alnico continued to be the industry standard for high-end permanent magnets. ${ }^{12}$

The next big breakthrough was $\mathrm{SmCo}_{5}$ in $1966 .^{13,14}$ This represented a new class of materials where the dominant force linking the direction of the magnetic flux to the body of the magnet is magnetocrystalline anisotropy $\left(K_{1}\right)$ rather than the shape of the magnetic particle (i.e., shape anisotropy).** Further work found better alloys in this class with the highest $(B H)_{\max }$ being the $\mathrm{Sm}_{2} \mathrm{TM}_{17}$ ( $\mathrm{TM}=$ transition metals, typically $\mathrm{Co}$ and $\mathrm{Fe}$ ) compound, but high Co content is required for use in commercial applications. ${ }^{15,16}$ The next big jump in energy density came in 1981, after the price for Co increased precipitously due to political unrest in what is now the Democratic Republic of the Congo, the world's leading producer for high-quality, lowcost cobalt. This uncertainty in the Co market spurred researchers in Japan and the United States to investigate alternatives, and it resulted in the discovery of a new compound based on $\mathrm{Nd}, \mathrm{Fe}$, and B. ${ }^{17-19}$ This stoichiometric, layered tetragonal compound $\left(\mathrm{Nd}_{2} \mathrm{Fe}_{14} \mathrm{~B}\right.$, space group $\left.\mathrm{P} 42 / \mathrm{m} \mathrm{n} \mathrm{m}\right)$ has a nearly ideal separation of the Fe atoms resulting in strong exchange coupling of the $\mathrm{Fe}$ and $\mathrm{Nd} \mathrm{su-}$ blattices resulting in the highest theoretical $(B H)_{\max }$ of any compound to date at 64 MGOe. This compound and alloys derived from it has enabled

\footnotetext{
**While PtCo was the first compound discovered with highmagnetocrystalline anisotropy, its cost rendered it impractical for commercial use in bulk.
} 


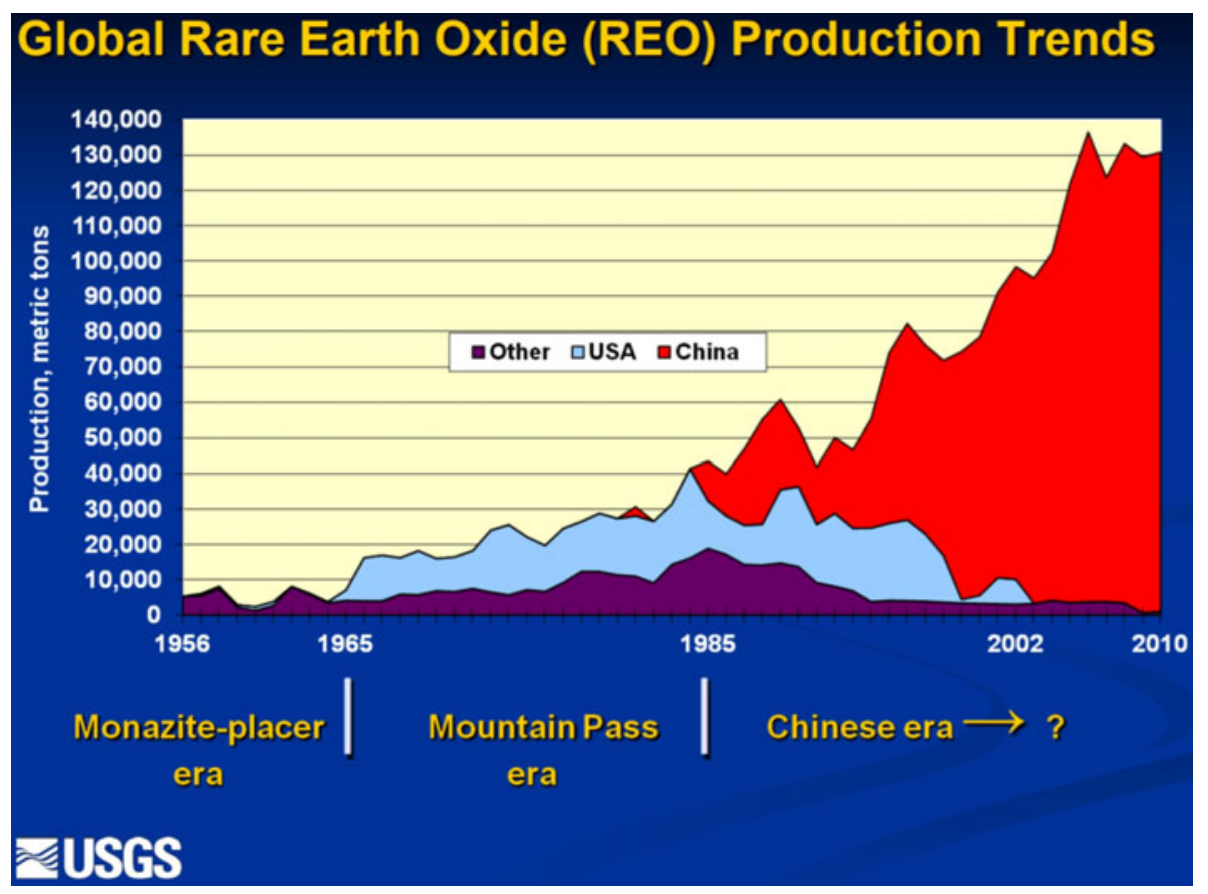

Fig. 2. Timeline showing the worldwide production of rare earth elements on an oxide basis by major producers ${ }^{7}$.

the miniaturization of many of the more common consumer products such as ear buds for portable audio devices and hard disk drives. The estimated market demand for Nd-based magnets in 2012 in disk drives, CD-ROMs, and DVD-ROMs alone is forecast to exceed 13,100 tons. ${ }^{\dagger}$

With the discovery of the rare earth elementbased magnet alloys, using primarily $\mathrm{Nd}$ and $\mathrm{Sm}$, the demand for rare earth element metals also increased throughout the 1980s. At the same time, new rare earth element resources were becoming available in China, causing a significant reduction in prices. The major producer in the world, the Molycorp's Mountain Pass mine in California, was experiencing problems with aging infrastructure, which required significant investment. With the low rare earth element prices, improvements to the Mountain Pass mine were not economically viable, and in a few short years in the 1990s, China became the main producer of rare earth elements (Fig. 2). ${ }^{7}$ By 2000, China was dominating the worldwide rare earth element production. During the discovery period of rare earth element-based magnets, the major producers of rare earth element-based magnet alloys were in Japan and the United States. Over the period 2001 to 2005 , the majority of the rare earth element-based magnet companies either ceased production or relocated to China to take advantage of lower manufacturing costs. Concurrently, numerous domestic Chinese manufacturers initiated the production of Nd-based magnets. While

${ }^{\dagger}$ Projected market demand is a compilation of industry and government sources. the demand for rare earth element-based magnets for disk drives, other electronics, and power hand tools had been increasing steadily, the rare earth supplies were more than capable of meeting that demand. Growing concern for air quality, carbon emissions, and rising cost of petroleum spurred the demand for alternatives to traditional production and for more efficient utilization of energy. This resulted in a rapid escalation of demand during the past decade for hybrid electric cars and wind-powered electric generators. This increased demand resulted in political pressures with regard to supply. In 2009, China announced that it would begin to curtail its export of the rare earths europium, terbium, and dysprosium. ${ }^{20,21}$ In March 2012, the European Union, Japan and United States announced that they would be filing a complaint for unfair trading practices against China's export quotas on rare earth elements to the World Trade Organization. $^{22}$

Regardless of the politics surrounding rare earth elements, increase in demand, driven primarily by electric machines, will be a major factor in both cost and availability of rare earth elements in the near future. The higher temperature performance requirement of generators and traction motors requires an adjustment to the Nd-based permanent magnets alloy to improve its high temperature resistance to demagnetization. This is accomplished with the addition of heavy rare earth elements, primarily Dy. Dy, while reducing $B_{\mathrm{r}}$, provides a moderate increase in $T_{\mathrm{c}}$ and a significant increase in the anisotropy field, which helps maintain a useful $H_{\text {cJ }}$ at temperatures up to $\sim 200^{\circ} \mathrm{C}$. The availability, 


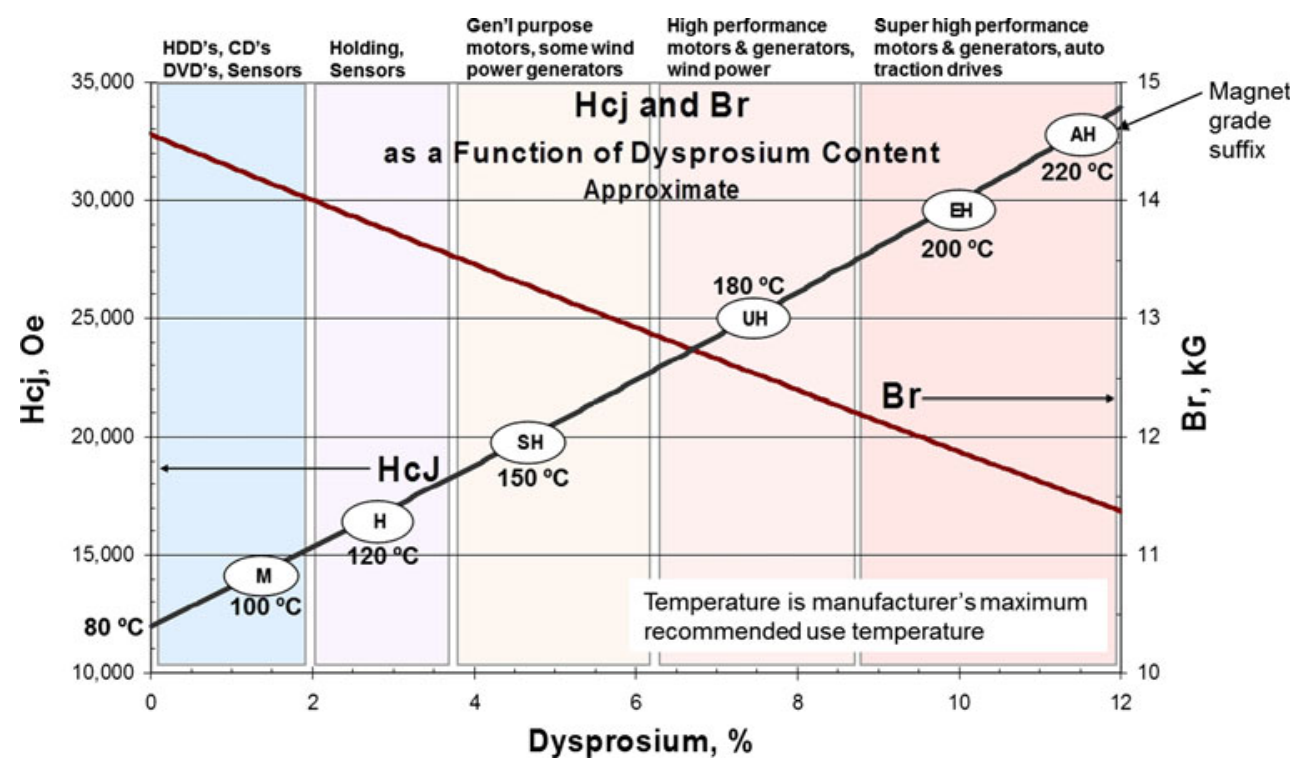

Fig. 3. A comparison of the Dy content (in wt.\% of the alloy) in various grades of Nd-based permanent magnets and how the Dy content affects the intrinsic coercivity $\left(H_{\mathrm{cJ}}\right)$ and induction $\left(B_{\mathrm{r}}\right)$. The general uses for the various grades of magnets is shown along the top columns. Data are supplied by Arnold Magnetic Technologies, www.arnoldmagnetics.com.

hence cost, of the rare earth elements is not uniform. Dy, being one of the more "rare" of the not-sorare rare earths, was trading near $\$ 100 / \mathrm{kg}$ in early 2009 to as high as $\$ 3400 / \mathrm{kg}$ in August of 2011, and it remains near $\$ 2000 / \mathrm{kg}$ as of April 2012. The amount of Dy added can be as high as 12 wt.\% for the more demanding electric machine applications performing at $220^{\circ} \mathrm{C}$ (Fig. 3). ${ }^{23}$ There appears to be sufficient resources for historical demands. However, projected demands for electric vehicles and wind turbines through the next 5-10 years shows that the availability of $\mathrm{Nd}$, while a concern is not as critical as Dy if Nd-based permanent magnets are used in these applications. Even with projected increase in rare earth element production from new mines coming online (Table I), this demand will not be met. Switching to Sm-based alloys does not alleviate the shortage of resources since Sm comprises less than $4 \%$ of the rare earth elements. Samarium is not available in adequate quantities to provide the volume of magnet product needed for a wholesale replacement of Nd-based magnets. It does represent a complementary product, especially for products expected to perform at temperatures above $160^{\circ} \mathrm{C}$. The trade-offs between rare earth elementbased magnets and potential substitutes will be discussed in more detail below.

Given the current state of technology, the options for replacing permanent magnet machines all have shortcomings. For wind turbines, the options are to continue to install induction-based generators or hybrid drive generators with a simpler 2-stage gearbox and $1 / 3$ the weight of permanent magnets required for a direct drive generator. For larger systems, $10 \mathrm{MW}$ and up, superconducting generators are being researched. For automotive traction drive motors, the size and weight considerations are more demanding, especially for hybrid vehicles due to limited space between the internal combustion engine and the wheels. While induction motors are an option for all electric vehicles, power conversion efficiency and mass-to-power ratio clearly favor a permanent magnet-based motor. ${ }^{2}$ However, the advantages of permanent magnet-based machines disappear if lower energy product non-rare earth element-based magnets are used. Even though most non-rare earth element permanent magnet materials developed prior to the invention of rare earth element permanent magnets are still commercially produced, the development of these older materials has been neglected. In this article, we will discuss a few of the more promising non-rare earth element systems that have the potential for replacing rare earth element-based permanent magnet alloys in electric machines and challenges to improving their properties to meet the demanding performance criteria for permanent magnet machines.

\section{ALTERNATIVE COMPOUNDS}

A high energy density is a necessary, but not a sufficient, condition for a commercially viable magnet alloy. It must be stable enough for the application so that the irreversible losses are minimal over the expected lifetime of the device, which can be 25 years for generators, and 10 or more years for traction drive motors. The intrinsic coercivity must be sufficient to resist demagnetization under maximum load and temperature. It must be made to be thermodynamically and physically stable (minimal flux change with temperature and resistance to corrosion, decomposition or oxidation) 
in the environment the device will operate. For instance, rare earth element-based alloys are prone to rapid breakdown in wet, warm, and corrosive environments if not adequately coated. ${ }^{24,25}$ Since the moments couple along specific crystallographic axes, it is necessary to grain-align the compound in order to attain the maximum energy product. But this texturing must be done to exacting tolerances. Ideally, the grains should be under the single domain limit, the size at which the energy of a domain wall in a grain exceeds the reduction in magnetostatic energy resulting from the domain wall. In practice, other factors that limit the nucleation of reverse domains and pin domain walls once the reverse domains nucleate allow the grain size to be about an order of magnitude larger. For ferrites, that is $\sim 1 \mu \mathrm{m}$ equivalent spherical diameter, $\sim 3 \mu \mathrm{m}$ for Nd-based magnets, and $4-5 \mu \mathrm{m}$ for SmCo. Highly irregular grain boundaries are also detrimental to domain-wall pinning, thus reducing coercivity. This extreme sensitivity to processing can greatly affect manufacturing costs. For instance, isotropic Nd-based magnets are produced by rapid quenching techniques resulting in grain sizes of a few tens of nanometers. These alloys are used in bonded magnets that have an energy product of $\sim 10$ MGOe while fully dense isotropic magnets can have energy products up to 16 MGOe and are less expensive to produce than sintered alloys. Figure 4 shows a scale of approximate cost as a function of maximum energy product for a wide range of permanent magnets. Since there are various grades and chemistries for many permanent magnet alloys, the boxes are approximate ranges of performance and cost. Part of the higher cost in the sintered rare earth element-based magnets is that powder metallurgical production results in large amounts of waste: grind swarf and saw kerf, as well as breakage. An additional concern for permanent magnetbased electric machines is the low electrical resistivity of the metal alloys that enhances eddy current losses unlike most permanent magnet ferrites, which are nonmetallic and nonconductive. Therefore, to minimize cost, the size and shape of the magnets must be closely matched to the motor design. Injection molding would accomplish this, but achieving the necessary energy density needed for generators and traction motors has been a challenge even in Nd-based magnets. This is due to the dilution effect of the nonmagnetic binder, which occupies about $35 \%$ of the magnet volume in injection molded magnets and 20-25\% in compression bonded magnets.

While a large maximum energy product does provide electric machine designers the greatest flexibility, there are considerations beyond $(B H)_{\max }$. These include the $T_{\mathrm{c}}$ (Curie temperature) and the temperature dependence of the magnetic properties (Fig. 5). In the following discussion, we will focus on those compounds that show some promising characteristics or pathways to improving the 


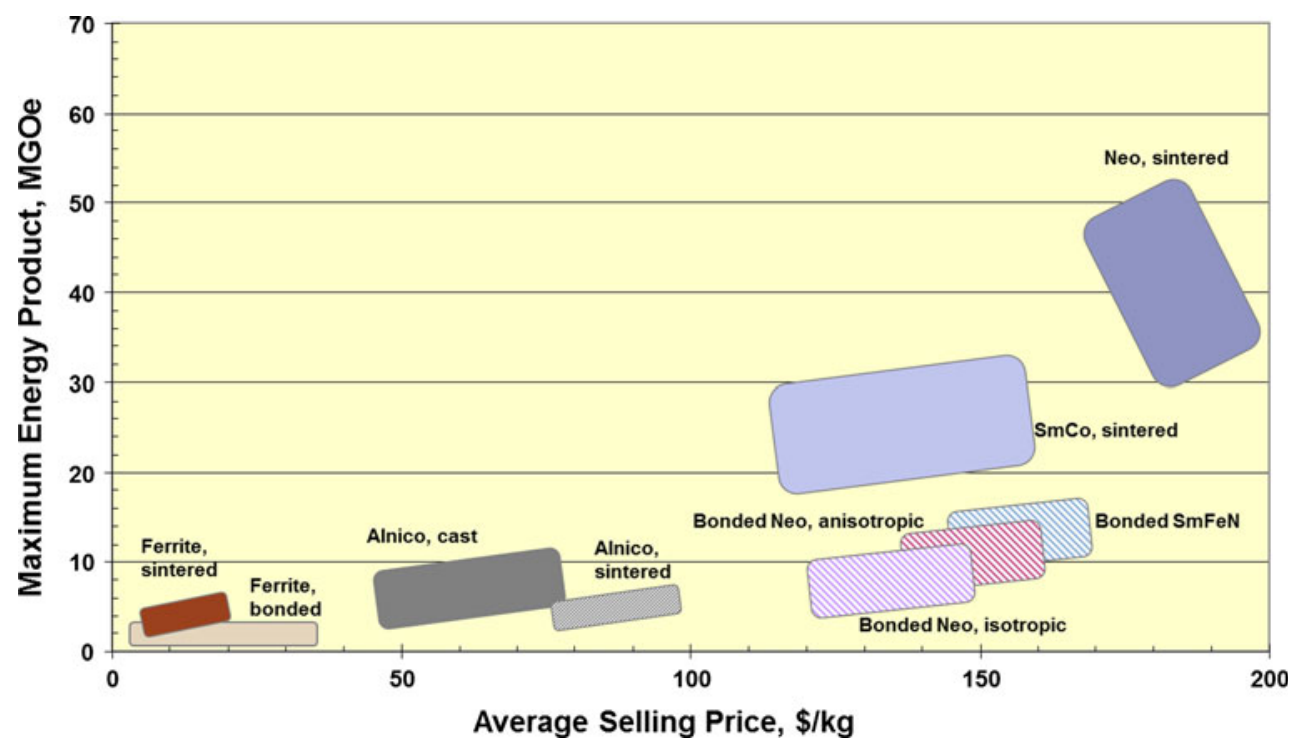

Fig. 4. A composite of the maximum energy product for various commercially produced permanent magnets and their relative costs. Many factors such as shape, complexity, and size contribute to a magnet's selling price. The values shown are fair estimates of standard shapes and sizes. The selling price is that observed in the United States and Europe.

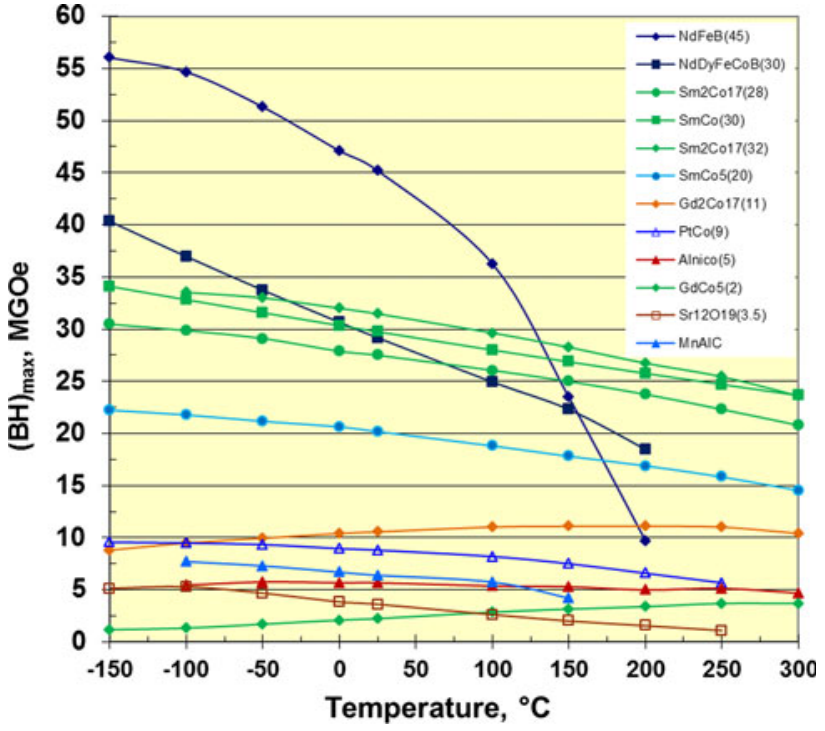

Fig. 5. The temperature performance of the maximum energy product as a function of temperature for many common commercial magnets and some promising alternatives. While the $\mathrm{Nd}$-based magnets have superior maximum energy product at $\sim 100^{\circ} \mathrm{C}$ or below, the Sm-based alloys do better above $200^{\circ} \mathrm{C}$. Currently, Dy is added to $\mathrm{Nd}$-based alloys to boost its performance in generators and traction motors. Many alternatives to Nd-based alloys will need to boost their maximum energy product by a factor or two to be comparable with current rare earth element-based magnets.

compounds' magnetic properties that would allow for use in generators and traction motors.

\section{Alnico}

Alnico has a number of very promising characteristics: a high $T_{\mathrm{c}}$, low thermal coefficient for coercivity and induction, and the ability to magnetize in nonplanar orientations. Significant strides in improving the energy density were made in the $1950 \mathrm{~s}$ and $1960 \mathrm{~s}$ based primarily on processing. ${ }^{26,27}$ The highest energy product alnico grades are 5-7 and 9 . These are both grain aligned and spinodally decomposed $^{28}$ as they are cooled in a magnetic field. ${ }^{29}$ The improvements in properties were based primarily on empirical studies. Computational and characterization tools to understand and predict optimal nanostructure of the spinodal domains were three decades in the future. A number of minor alloying elements, primarily $\mathrm{Cu}, \mathrm{Ti}$, and $\mathrm{Nb}$, are added to the base alloy of 8-13 wt.\% Al, 13-28 wt.\% $\mathrm{Ni}, 0-42$ wt.\% Co, with the balance Fe, to promote columnar growth and enhance $H_{\mathrm{cJ}}$. Key questions remaining to be addressed in further optimization of this class of alloys include: To what extent has the spinodal decomposition been optimized? More specifically, how does the dimension, uniformity of shape, and degree of chemical segregation affect $H_{\mathrm{cJ}}$ ? For example, doubling of the $H_{\mathrm{c}}$ of alnico 5 to 7 from 750 to 1500 oersteds without sacrificing $B_{\mathrm{r}}$ would result in an increase in $(B H)_{\max }$ from 7.5 to $\sim 14$ MGOe. Determining how to achieve this without a better understanding of the fundamental mechanisms for domain pinning in these compounds would be difficult.

\section{Ferrites}

By weight, ferrites are by far the largest single class of permanent magnets produced (567,330 tons in 2010). They are made with inexpensive elements and, being oxides, have excellent chemical stability for electric machine applications. While significant improvements have been made in preceding decades 
in the energy density for ferrites, ${ }^{30}$ there are a number of significant intrinsic limitations for this class of compounds. First, they have a very high mass-to-magnetization ratio so that the size of a permanent magnet-based electric machine would be too large for a hybrid vehicle. For wind turbine applications, the cost of the additional structure to support the more massive nacelle is more than the incremental cost of the rare earth element permanent magnets. The low magnetization is due to the fact the ferrites are actually ferrimagnets with two different $\mathrm{Fe}$ lattices with unequal magnetizations that are coupled antiferromagnetically. Therefore, the net magnetization is the difference between the two lattice magnetizations rather than the sum. As a result, the magnetization is approximately a factor of 4 less than that of $\mathrm{Nd}$-based permanent magnets. Unlike Nd-based permanent magnets, the low temperature performance is a problem. The two magnetic sublattices have different temperature dependences resulting in large temperature dependences of the magnetic properties. In the ferrites, this results in the net magnetization increasing much more rapidly than the anisotropy constant with decreasing temperature. The anisotropy field and, hence, the coercivity is determined by the ratio of these two with the net result that $H_{\text {ci }}$ decreases with decreasing temperature, a unique property of these materials. At $-40^{\circ} \mathrm{C}$, the coercivity is reduced to the minimum practical value, making electric machines based on these materials marginally inoperable in higher latitudes during the coldest part of the winter. Significant improvements in $B_{\mathrm{r}}$ are difficult to achieve simply because the magnetic coupling is based on antiferromagnetic exchange between the magnetic sublattices across oxygen atoms. Doping of selective $\mathrm{Fe}$ sites with Co has resulted in significant enhancements of $B_{\mathrm{r}}$ and $H_{\mathrm{cJ}}$ when paired with La substitution on the Sr site, but the extent of doping is limited by phase stability. While the magnetization can be increased by lowering the magnetization of the sublattice with the lower magnetization, this inevitably decreases the exchange and lowers $T_{\mathrm{c}}$.

\section{Other Fe-Based Compounds Without Rare Earth Elements}

The challenge to designing a high-performance permanent magnet is getting enough density of the elements with significant moments, the transition elements (TM) $\mathrm{Mn}, \mathrm{Cr}, \mathrm{Fe}$ and $\mathrm{Ni}$, in a configuration where their moments can align ferromagnetically. $\mathrm{Fe}$ is preferred since it has the largest moment, is abundant, and is inexpensive. Unfortunately, there is an optimum TM-TM spacing for each element; closer spacing tends toward antiferromagnetic alignment while larger spacings reduce the exchange and lowers $T_{\mathrm{c}}$ and the volume magnetization. The optimum spacing is achieved using nonmagnetic elements to stabilize suitable structures.
Additionally, the compound structure should form a lower symmetry compound than cubic, which would have the additional benefit of large magnetocrystalline anisotropy to provide coercivity. In fact, FePt forming the L10 structure is an ideal compound, except that cost and availability of $\mathrm{Pt}$ renders it impractical for any high-volume application.

TM compounds with $\mathrm{N}$ have shown some promise $^{31-33}$ as they have a large moment and have the potential to be produced in volume at low cost. The main challenge has been that the magnetic phases of interest are either metastable compounds or have a moderately low decomposition temperatures, ${ }^{34-36}$ and these phases have yet to demonstrate significant coercivity. ${ }^{37}$ There are two metastable compounds of interest, the tetragonal $\alpha^{\prime}$ with about 10 at. $\% \mathrm{~N}$ and the more ordered $\alpha^{\prime \prime} \mathrm{Fe}_{16} \mathrm{~N}_{2} \cdot{ }^{38}$ For instance, nitriding $\mathrm{Sm}_{2} \mathrm{Fe}_{17}$ provides a high-energy product compound but has yet to be fabricated into an oriented, fully dense material. ${ }^{31,39}$ Compounds with even moderate decomposition temperatures (in the range of $\sim 400^{\circ} \mathrm{C}$ ) have two significant drawbacks for electric machines; first, they may have unacceptable irreversible losses over time, and more importantly, in order to obtain high energy density in a magnet, the magnet must approach $100 \%$ density. Therefore, being able to synthesize fine powders with a theoretical $(B H)_{\max }$ comparable with Nd-based alloys is not sufficient. These compounds will need to be compacted into grain-aligned, dense monolithic parts. Typical processing conditions for consolidation of powders into dense compacts results in decomposing the compound or in excessive grain growth, either of which significantly degrades magnetic performance. The challenge to producing usable permanent magnets out of this class of alloys will be to stabilize their metastable structures so that they can be consolidated into dense monolithic parts with long-term stability. ${ }^{40}$

The search for new or modified materials may be aided by modern computer codes based on density functional theory (DFT). These allow for a fairly rapid assessment of "what if" scenarios for either known or postulated compounds. ${ }^{41}$ This can be as straightforward as evaluating substitutions to known compounds and determining their thermodynamic stability or as sophisticated as performing genetic searches for new compounds. ${ }^{42}$ Critical metrics for promising compounds are the significant number of ferromagnetic moments and high magnetocrystalline anisotropy. ${ }^{43}$ Since magnetocrystalline anisotropy is a combination of the spin-orbit and crystal field effects, these are all parameters that can be calculated using a number of DFT programs. ${ }^{44,45}$ Small concentrations of impurities of C and $\mathrm{N}$ in bcc Fe were shown to modify the hybridization of the $\mathrm{Fe} 3 \mathrm{~d}$ orbitals, even though the anisotropy contribution of the $\mathrm{C}$ and $\mathrm{N}$ were small. $^{46-48}$ More important could be the strain effects from the interstitials themselves. Theoretical calculations have suggested that compounds 
containing mixtures of $3 \mathrm{~d}$ transition elements and $4 \mathrm{~d}$ and $5 \mathrm{~d}$ elements can have large magnetocrystalline anisotropy via large spin-orbit coupling. ${ }^{49,50}$

One interesting approach is to look at marginally stable materials. Fe-Ni meteorites contain a magnetically hard FeNi compound having the L10 structure, called tetrataenite. ${ }^{51}$ This compound has a relatively high magnetocrystalline anisotropy and a magnetization equal to that of $\mathrm{Nd}_{2} \mathrm{Fe}_{14} \mathrm{~B}$. Unfortunately, these phases do not form under normal laboratory conditions but rather require the extremely slow cooling rates which have occurred in meteorites. The challenge is to develop a kinetic pathway to form the hard magnetic FeNi L10 structure. The existence of this phase suggests that all the possible permutations of Fe-based systems have not been fully exploited. Thermodynamic calculations, more in-depth first-principles studies and more comprehensive screen tools, such as combinatorial analysis, will be required to more fully investigate this phase space. Advanced far-fromequilibrium processing will be needed to explore new phase space for compounds that possess anisotropy with reasonable magnetization by chemical substitutions and other processing methods to induce chemical ordering.

\section{Mn-Based Compounds}

There are two possible non-Fe-based compounds that have shown significant coercivity: $\mathrm{MnBi}$ and MnAl. While Mn-containing compounds are typically antiferromagnetic, the moments can be induced to align if the separation of the Mn atoms is greater than $2.96 \AA .{ }^{52}$ However, the total moments in these compounds tend to be low as the large spacing translates to a small volume fraction of magnetic atoms which results in a low energy density. $\mathrm{MnBi}$ is unusual in that its coercivity actually increases with temperature. Its $T_{\mathrm{c}}$ is limited by the decomposition of the magnetic phase through a peritectic reaction at $355^{\circ} \mathrm{C} .^{53}$ Reported $(B H)_{\max }$ is in the range of $\sim 6-7$ MGOe ${ }^{54}$ The magnetic phase is hexagonal, having the NiAs structure. ${ }^{55}$ The low melting temperature of $\mathrm{Bi}$ and the high vapor pressure of Mn have posed some challenges to processing, but the compound can be formed using rapid solidification. ${ }^{56}$ However, this results in an isotropic structure greatly reducing the energy product. Like most permanent magnet compounds, maintaining a fine grain structure is crucial to retaining its coercivity.

MnAl: With a theoretical $(\mathrm{BH})_{\max }$ of $\sim 12$ MGOe and with its low density of $5.2 \mathrm{~g} / \mathrm{cm}^{3}, \mathrm{MnAl}$ has an attractive energy density per unit mass. ${ }^{57-59}$ The metastable ferromagnetic phase is of the L10-type and termed the $\tau$ phase. It is difficult to synthesize due to its sluggish reactions at low temperatures where it is stable. ${ }^{52,60}$ Mechanical milling has been shown to be a viable route to producing this metastable phase while retaining a fine grain structure.
As with rapid solidification, this method results in an isotropic grain structure reducing the magnetic properties. While the addition of $\mathrm{C}$ increases the stability of the $\tau$ phase, it significantly decreases the anisotropy field $(55-39 \mathrm{kOe})$ and $T_{\mathrm{c}}\left(380-285^{\circ} \mathrm{C}\right){ }^{61}$ This lower $T_{\mathrm{c}}$ greatly reduces the potential for energy applications.

The challenge to both of these systems is to increase the operating temperature capability and magnetization. Increasing the operating temperature in $\mathrm{MnBi}$ is particularly problematic since it is the stability of the phase rather than the strength of the exchange interaction that limits high-temperature operation. Thus, a large temperature excursion would not only demagnetize the magnet but also result in the formation of a liquid phase within the machine. For most generators and traction motors, the operating temperature is limited to well below $355^{\circ} \mathrm{C}$ by the temperature stability of other components, in particular the insulating materials, so $355^{\circ} \mathrm{C}$ is not an engineering limit. However, ternary additions that could possibly increase $M_{\mathrm{s}}$ tend to reduce the peritectic temperature, limiting choices for alloy improvement. ${ }^{62,63}$ On the plus side, the coercive field for $\mathrm{MnBi}$ is nearly five times that of Nd-based compounds at $127^{\circ} \mathrm{C} .{ }^{64}$ The temperature stability of the MnAl $\tau$-phase is also of concern since prolonged annealing has been shown to decompose the binary phase. ${ }^{65}$ While additions of $\mathrm{C}$ can stabilize the magnetic phase, the resulting degradation to the magnetic properties is unacceptable. Finding an alloying element that increases the stability of the $\tau$-phase without degrading the magnetic properties will be the key making this a viable permanent magnet.

\section{Nonstrategic Rare Earth Elements}

The resource availability and demand for the various rare earth elements are not correlated; hence, the strategic concern is not uniform across the Lanthanide series. Only a few of the rare earth elements currently have high market demand, both in absolute terms and more importantly, relative to their natural abundances in commercially viable mines. The most critical supply risks in the next 5 years are $\mathrm{Dy}, \mathrm{Eu}, \mathrm{Nd}, \mathrm{Tb}$, and $\mathrm{Y}$ while $\mathrm{Ce}, \mathrm{La}$, and to some extent $\mathrm{Pr}$ are less so. ${ }^{6}$ In terms of resources, Ce comprises nearly $40 \%$ of all rare earth elements in the Earth's crust, ${ }^{7}$ and in light rare earth element deposits like Mountain Pass, the ratio can be as high as $50 \%$. Conversely, Dy, which is critical to providing the increase in high temperature capability for Nd-based alloys, is less than $2 \%$ of all the rare earth elements and is found in abundance in only a few localities. While a wide variety of magnetic compounds can be synthesized from nearly every lanthanide element, few have the high energy density of the Nd-, Pr-, and Sm-based compounds. Heavy rare earths, for instance, are unsuitable for permanent magnets even though they have large 
moments since these moments couple antiparallel to the transition-metal sublattice. Furthermore, the rare earth anisotropy contribution (easy-axis or easy-plane) changes sign in the middle of each of the two rare earth half-series. This means, for example, that $\mathrm{Sm}$ yields a positive anisotropy contribution but not Nd. ${ }^{12}$ Furthermore, the de Gennes factor causes the rare earth elements from the middle of the series to yield the largest Curie temperature. The $T_{\mathrm{c}}$ for $\mathrm{Gd}_{2} \mathrm{Fe}_{14} \mathrm{~B}$ is nearly $100^{\circ} \mathrm{C}$ higher than $\mathrm{Nd}_{2} \mathrm{Fe}_{14} \mathrm{~B}$ but its anisotropy is too low to make is it a useful magnet. ${ }^{66}$ Substitutions, even at low levels, can have a profound effect on the magnetic properties. ${ }^{66,67}$ While considerable efforts have gone into exploring a large number of possible minor alloying elements, the majority of the work has been in Ndand Sm-based compounds. Much less research has been performed on compounds based on the more abundant $\mathrm{Ce}$ and $\mathrm{La}$. Ce and La comprise up to about $85 \%$ of the ore in most light rare earth mines on an oxide basis. Alloys which utilize some of these more abundant rare earth elements would help to mitigate excessive demand on $\mathrm{Nd}$ and more importantly Dy, and at the same time increase market demand for the more abundant Ce and La providing a higher return on investment for the light rare earth element mines. However, La does not have a magnetic moment and, hence, functions only as a spacer in the crystal structure, and Ce is not well behaved due to its unusual electronic structure. Unfortunately, in intermetallic compounds with $\mathrm{Fe}$, $\mathrm{Co}$, or $\mathrm{Ni}$, Ce has a strong tendency to give up its single $4 \mathrm{f}$ electron and become a $4+$ or a mixed valence ion rather than the $3+$ typical of most of the other rare earths. While $4+$ Ce might be expected to behave like the nonmagnetic rare earth elements $\mathrm{La}, \mathrm{Lu}$, and $\mathrm{Y}$ with little effect on the $T_{\mathrm{c}}$ and only having magnetocrystalline anisotropy from the TM lattice, it appears that Ce is mixed valence with a resulting hybridization of the $4 \mathrm{f}$ electrons with the transition metal $3 \mathrm{~d}$ electrons, which results in the lowering of $T_{\mathrm{c}}$. If means can be found to compensate for the detrimental properties of $\mathrm{Ce}$, then the availability of rare earth element magnets from existing sources could be greatly enhanced.

\section{DISCUSSION}

While a large number of permanent magnet compounds has been discovered over the last 50 years, ${ }^{68}$ the number that have the necessary characteristics to be viable candidates for the demanding application of generators and traction motors is limited. The key factors that need to be considered when investigating new or modified compositions are $T_{\mathrm{c}}, K_{1}$, and $M_{\mathrm{s}}$. For generators and traction motors, the weight-to-power ratio is of prime importance. While Curie temperature in and of itself is not that important provided it is above the operating window for the electric machines, it places an absolute limit on the temperature dependence of $B_{\mathrm{r}}$ and $H_{\mathrm{cJ}}$. Large negative temperature coefficients for these parameters are problematic. First, it requires that considerably more material be used than that required based on normal operating temperatures since the motor must be designed to maximum load conditions. Alternatively, additional cooling systems must be employed. Second, power control is more complicated since motor power output is not linear with temperature. Operating too close to the minimum load line for the motor can also result in large irreversible losses, reducing motor lifetime. The magnetocrystalline anisotropy also provides a key metric to the ultimate $H_{\mathrm{cJ}}$ that can be achieved in the compound while $M_{\mathrm{s}}$ is a metric for the $B_{\mathrm{r}}$. How closely a magnet based on a given compound can achieve these theoretical limits is dependent on processing. Here, it is well established that the grain size must approach the single domain particle size limit where the formation of a domain wall inside the grain is unfavorable. Since the total magnetization of a magnet is the vector sum of the individual grains along the direction of the bulk magnetization, the degree of grain alignment has a significant role to play. In fact, the maximum energy product of an aligned magnet is 2.5 times that of an isotropic magnet. In addition, irregularities at grain boundaries can also provide regions of high flux concentration and reduce the area of the domain wall required to nucleate a reverse domain. This can result in easy nucleation of reversal of magnetic domains. Therefore, being able to reproducibly process new materials to such exacting parameters sets a high bar to introduce new materials into the market.

In the $\mathrm{Nd}_{2} \mathrm{Fe}_{14} \mathrm{~B}$ magnet, there are two functional parts of the structure: the Fe layers provide the magnetization and $T_{\mathrm{c}}$ while the Nd layers provide the magnetocrystalline anisotropy. At room temperature, there is more anisotropy than is actually required for most applications, but the compound lacks sufficient moments. Additional moments can be provided by nanostructuring of a high-magnetization soft phase and a high-anisotropy hard phase. ${ }^{69}$ Here, the mixture of a hard and soft magnetic phase at the appropriate length scales, typically on the order of a few $\mathrm{nm}$, gives rise to uniform magnetic response as a single domain extends over both hard and soft phases providing a high magnetization while their small dimension limits easy reversal of the soft phase since their moments are pinned to the hard phase. While modeling has suggested that energy products of such nanostructured alloys could exceed 100 MGOe in an anisotropic magnet, nothing close to this has been achieved in practice. $^{70}$ The primary difficulty is that processing methods which provide good texturing tend to require high temperatures, which result in coarser grained alloys than required for effective exchange coupling. While this is being actively pursued in the rare earth element-based compounds, ${ }^{39}$ this 
approach is just as valid for other ferromagnetic compounds and is particularly attractive for $\mathrm{Mn}$ based alloys, which have high $H_{\mathrm{cJ}}$ but poor $B_{\mathrm{r}}$.

Compounds based primarily on $\mathrm{Fe}$ have the opposite problem. Here, the challenge will be to induce $H_{\mathrm{cJ}}$ without sacrificing $B_{\mathrm{r}}$. For most of the Fe-based magnets, $H_{\mathrm{cJ}}$ is dependent on shape rather than magnetocrystalline anisotropy. For those compounds utilizing shape anisotropy, such as alnico, the key factor is how to improve the shape anisotropy. Theoretical studies suggest that increasing the aspect ratio will increase $H_{\mathrm{cJ} .}{ }^{71}$ Recent calculations also suggest that the sharpness of the interface between the magnetic and nonmagnetic phase can also have a measurable effect. Another promising approach discussed above is to induce magnetocrystalline anisotropy. The most promising approach is to judiciously introduce either interstitials or substitutional atoms that result in degeneracy of the normal bcc Fe structure, as seen in the $\mathrm{Fe}-\mathrm{N}$ compounds. These substitutions need to be thermodynamically stable and easily produced on industrial scales. However, while nanostructuring is an excellent pathway to produce novel metastable compounds, engineering materials must be near theoretical density to be economical.

For a mass market, the materials of choice for the alloy development must be plentiful, secure, and relatively inexpensive. The electric vehicle market is expected to grow to 20 million vehicles per year, or approximately $20 \%$ of the global vehicle market by 2020 . Current technology uses $12.3-13.3 \mathrm{~g}$ of rare earth per $\mathrm{kW}$ for motors with peak power 60 and $110 \mathrm{~kW} .{ }^{72}$ The increased demand for rare earth elements can be estimated to be between 6400 and 11,200 metric tons of which $\sim 25$ wt.\% is Dy. Current direct drive wind generators utilize approximately $600 \mathrm{~kg}$ of Nd-magnets per MW. In 2010, China installed 18,928 MW of wind power generation or approximately $50 \%$ of all wind power installed globally that year. Of that total, $\sim 25 \%$ was permanent magnet generators using 2840 metric tons of Nd-magnets. The rest of the world's wind generators up to this point have been primarily induction machines, and the small number of permanent magnet generators used only 751 metric tons of $\mathrm{Nd}$ magnets. If the rest of the world were to install permanent magnet generators to meet greenhouse gas reduction, the demand for $\mathrm{Nd}$ magnets would increase by nearly $50 \%$ by 2020 from 2010 levels even while assuming about a 30\% increase in the efficiency of the newer generators and that only $75 \%$ newly installed capacity would use permanent magnet generators. However, these numbers belie the real problem, which is an adequate supply Dy (Table II). Of particular importance is the increased demand for Dy relative to its natural abundance. While demand for the $\mathrm{Nd}$ is growing rapidly under the most aggressive scenario for permanent magnet-based electric machines, increased production will generally keep pace (Table I). The most significant bottleneck in supplying higher demand for permanent magnet generators and traction motors is the upper limit temperature performance and the associated cost and availability of the Dy. It is here where the technological breakthroughs are going to be most important.

While the Nd-based magnets possess the highest energy product near room temperature and down to $140 \mathrm{~K}$, at higher temperatures, especially the upper operating temperature range for hybrid vehicle motors and wind turbines, a number of other alloys are close contenders (Fig. 5). For instance, one of the advantages of alnico alloys is that they have a very flat energy density over a broad range of temperatures. While alnico's Co content is high and the availability of Co can also be a concern, the resources for Co are better distributed than rare earth elements. A modest gain in the energy density can make this alloy a viable alternative. Prospects for other $\mathrm{Fe}$ plus transition metal systems are longer term. While theoretical models have shown promise for this general class of alloys, it is not clear yet whether they can be synthesized and if so, would they have the thermodynamic stability to be used in a permanent magnet electric machines. The potential payback for an inexpensive high energy density

Table II. Projection of Dy supply and demand, in tons, and looming shortage based on projected increased demand for Nd-based permanent magnets for traction motors and wind generators

\begin{tabular}{|c|c|c|c|c|c|c|c|c|}
\hline & 2005 & 2010 & 2011 & 2012 & 2013 & 2014 & 2015 & 2020 \\
\hline Demand & 1015 & 1597 & 1836 & 2020 & 2181 & 2356 & 2544 & 3568 \\
\hline Shortage & & & -226 & -395 & -531 & -681 & -844 & \\
\hline \multicolumn{9}{|l|}{ Metal } \\
\hline Demand & 810 & 1280 & 1470 & 1620 & 1750 & 1880 & 2040 & 2850 \\
\hline
\end{tabular}

Assume $80 \%$ of the oxide is made into metal. 
magnet made from relatively abundant materials would be revolutionary.

Mn-based alloys also have promising high-temperature magnetic properties, in particular the $\mathrm{MnBi}$ class of compounds. These compounds pose a different set of materials optimization problems since they lack significant magnetization. While theoretical pathways have been identified with which to dramatically enhance magnetization in such compounds, it has yet to be demonstrated in a bulk compound.

\section{CONCLUSIONS}

The push to reduce greenhouse gasses will require a restructuring of both energy production and utilization. Electric machines for both power generation and traction motors can figure prominently in this new economy. While Nd-based permanent magnet electric machines provide obvious power-toweight advantage over induction machines, the availability and price of the rare earth metals are making permanent magnet-based electric machines less favorable. Of particular concern is the cost and availability of Dy, which is a key rare element with limited availability that is required to improve the high-temperature performance of Nd-based alloys for use in generators and traction motors. Alternatives to rare earth-based alloys do exist, but at the current time, they lack the necessary energy density to replace Nd-based magnets. Some of these potential replacements actually exceed performance of the Nd-based alloys above $200^{\circ} \mathrm{C}$. Many of these compounds have been known for decades, but serious interest in their optimization waned once the rare earth element-based compounds were discovered. Fortunately, researchers have new tools with which to characterize and understand the mechanisms for coercivity and magnetization in these compounds. Computational tools can provide detailed descriptions of the relationship between atomic composition, order, and interaction of the magnetic spins. These can even be used to discover potentially new compounds. Experimental methods for exploring wide areas of phase space are also being utilized to identify new magnetic compounds. Significant research efforts are underway to exploit these possibilities. Like the early 1980 s, a shortage of critical materials today can be a boon for materials discovery.

\section{ACKNOWLEDGEMENTS}

We would like to thank Ralph Skomski, David Sellmyer, and Jeff Shield for their helpful suggestions. This work was supported by the U.S. Department of Energy, Office of Energy Efficiency and Renewable Energy (EERE), under its Vehicle Technologies Program, through the Ames Laboratory. Ames Laboratory is operated by Iowa State University under contract DE-AC02-07CH11358.

\section{OPEN ACCESS}

This article is distributed under the terms of the Creative Commons Attribution License which permits any use, distribution, and reproduction in any medium, provided the original author(s) and the source are credited.

\section{REFERENCES}

1. Center for Transportation Analysis, U.S. Department of Energy, Transportation Energy Data Book, 30th ed. (Washington, DC: Center for Transportation Analysis, U.S. Department of Energy, 2011), http://cta.ornl.gov/data/tedb30/ Edition30_Full_Doc.pdf.

2. K.R. Weeber, M.R. Shah, K. Sivasubramaniam, A. El-Refaie, Q. Ronghai, C. Stephens, and S. Galioto, IEEE Power and Energy Society General Meeting, 2010, pp. 1-6.

3. A.M. Bradshaw and T. Hamacher, Chemsuschem 5, 550 (2012).

4. E. Peeters, J. Van Bael, and P. Van Tichelen, Proc. 2nd IASME/WSEAS International Conference on Energy \& Environment (www.worldses.org: WSEAS, 2007), pp. 154158.

5. Critical Materials Strategy, U.S. DOE, 2010, http://energy. gov/sites/prod/files/edg/news/documents/criticalmaterials strategy.pdf.

6. Critical Materials Strategy, U.S. DOE, 2011, http://energy. gov/sites/prod/files/DOE_CMS2011_FINAL_Full.pdf.

7. U.S. Geological Survey, Rare Earth Elements-Critical Resources for High Technology, USGS Fact Sheet 087-02, 2002.

8. M. Humphries, Rare Earth Elements: The Global Supply Chain (Washington, DC: C.R. Service, 2011).

9. R. Moss, E. Tzimas, H. Kara, P. Willis, and J. Kooroshy, Critical Metals in Strategic Energy Technologies-Assessing Rare Metals as Supply-Chain Bottlenecks in Low-Carbon Energy Technologies (Brussels, Belgium: Joint Research Center, Institute for Transuranium Elements, 2011), http:// publications.jrc.ec.europa.eu/repository/handle/111111111/ 22726.

10. H.P. Schlemmer, Radiologe 45, 356 (2005).

11. A.J.J. Koch, M.G. Van der Steeg, and K.J. de Vos, AIEE Spec. Publ. T-91, 173 (1957).

12. R. Skomski and J.M.D. Coey, Permanent Magnetism (Bristol, U.K: Institute of Physics, 1999).

13. W. Ostertag and K.J. Strnat, Acta Crystallogr. 21, 560 (1966).

14. K. Strnat, G. Hoffer, W. Ostertag, and J.C. Olson, J. Appl. Phys. 37, 1252 (1966).

15. M.G. Benz and D.L. Martin, Appl. Phys. Lett. 17, 176 (1970).

16. K.J. Strnat, Proc. IEEE 78, 923 (1990).

17. N.C. Koon, C.M. Williams, and B.N. Das, J. Appl. Phys. 52, 2535 (1981).

18. J.J. Croat, B Am. Phys. Soc. 26, 239 (1981).

19. M. Sagawa, W. Yamagishi, and Z. Henmi, J. Appl. Phys. 52, 2520 (1981).

20. P.C. Dent, Adv. Mater. Process. 16, 277 (2009).

21. D. Normile, Science 330, 1598 (2010).

22. T. Orlik and C.-W. Yap, "China's Rare Earth Recoil," Wall Street Journal (14 March 2012). http://online.wsj.com/ article/SB10001424052702304450004577278981461660606. html.

23. S. Constantinides (Paper presented at DOE Vehicle Technologies Program APEEM R\&D FY12 Kickoff Meeting, November 2-4, 2011, Oak Ridge National Laboratory, Oak Ridge, TN, 2011).

24. M. Katter, L. Zapf, R. Blank, W. Fernengel, and W. Rodewald, IEEE Trans. Magn. 37, 2474 (2001).

25. H. Bala, N.M. Trepak, S. Szymura, A.A. Lukin, V.A. Gaudyn, L.A. Isaicheva, G. Pawlowska, and L.A. Ilina, Intermetallics 9, 515 (2001).

26. R.B. Campbell and C.A. Julien, J. Appl. Phys. 32, S192 (1961). 
27. C.A. Julien and F.G. Jones, J. Appl. Phys. 36, 1173 (1965).

28. J.W. Cahn, Acta Metall. Mater. 9, 795 (1961).

29. J.W. Cahn, J. Appl. Phys. 34, 3581 (1963).

30. V.A.M. Brabers and K.H. Buschow, ed., Handbook of Magnetic Materials, vol. 8 (Amsterdam, the Netherlands: NorthHolland, 1995), p. 189.

31. J.M.D. Coey and P.A.I. Smith, J. Magn. Magn. Mater. 200, 405 (1999).

32. N. Ji, M.S. Osofsky, V. Lauter, L.F. Allard, X. Li, K.L. Jensen, H. Ambaye, E. Lara-Curzio, and J.P. Wang, Appl. Phys. Lett. 98, 092506 (2011). doi:10.1063/1.3560051.

33. N. Ji, L. Allard, E. Lara Curzio, and J.-P. Wang, Appl. Phys. Lett. 98, 092506 (2011).

34. K.H. Jack, Proc. R. Soc. Lond. Ser.-A 208, 200 (1951).

35. K.H. Jack, Proc. R. Soc. Lond. Ser.-A 208, 216 (1951).

36. B.J. Kooi, M.A.J. Somers, and E.J. Mittemeijer, Metall. Mater. Trans. A 27, 1063 (1996).

37. H. Takahashi, H. Shoji, and M. Takahashi, J. Magn. Magn. Mater. 174, 57 (1997)

38. M.Q. Huang, W.E. Wallace, S. Simizu, and S.G. Sankar, J. Magn. Magn. Mater. 135, 226 (1994).

39. G.C. Hadjipanayis, J.F. Liu, A. Gabay, and M. Marinescu, J. Iron. Steel Res. Int. 13, 12 (2006).

40. K.H. Jack, Nitrides Oxynit. 325-3, 91 (2000).

41. J. Hafner, C. Wolverton, and G. Ceder, MRS Bull. 31, 659 (2006).

42. M.C. Nguyen, X. Zhao, M. Ji, C.-Z. Wang, B. Harmon, and K.-M. Ho, J. Appl. Phys. 111, 07E338 (2012).

43. R. Skomski, A. Kashyap, and A. Enders, J. Appl. Phys. 109, 07E143 (2011). doi:10.1063/1.3562445.

44. K.D. Belashchenko, V.P. Antropov, and N.E. Zein, Phys. Rev. B 73, 073105 (2006). doi:10.1103/Physrevb.73.073105.

45. V.P. Antropov, M. van Schilfgaarde, S. Brink, and J.L. Xu, J. Appl. Phys. 99, 08F507 (2006).

46. N.I. Medvedeva, D. Van Aken, and J.E. Medvedeva, J. Phys.-Condens. Mat. 22, 316002 (2010). doi:10.1088/ 0953-8984/22/31/316002.

47. G. Rahman, I.G. Kim, H.K.D.H. Bhadeshia, and A.J. Freeman, Phys. Rev. B 81, 184423 (2010). doi:10.1103/Physrevb. 81.184423

48. M. Kogachi, N. Tadachi, H. Kohata, and H. Ishibashi, Intermetallics 13, 535 (2005).

49. A. Kashyap, P. Manchanda, P.K. Sahota, R. Skomski, J.E. Shield, and D.J. Sellmyer, IEEE Trans. Magn. 47, 3336 (2011).

50. V. Sharma, P. Manchanda, R. Skomski, D.J. Sellmyer, and A. Kashyap, J. Appl. Phys. 109, 07A727 (2011). doi:10.1063/ 1.3562256 .
51. E.R.D. Scott and R.S. Clarke, Nature 287, 255 (1980).

52. T. Ohtani, N. Kato, S. Kojima, K. Kojima, Y. Sakamoto, I. Konno, M. Tsukahara, and T. Kubo, IEEE Trans. Magn. 13, 1328 (1977).

53. H. Okamoto, Binary Alloy Phase Diagrams, ed. T.B. Massalski (Materials Park, OH: ASM, 1990).

54. D.T. Zhang, S. Cao, M. Yue, W.Q. Liu, J.X. Zhang, and Y. Qiang, J. Appl. Phys. 109, 07A722 (2011). doi:10.1063/1.35 61784 .

55. J.B. Yang, K. Kamaraju, W.B. Yelon, W.J. James, Q. Cai, and A. Bollero, Appl. Phys. Lett. 79, 1846 (2001).

56. X. Guo, X. Chen, Z. Altounian, and J.O. Stromolsen, Phys. Rev. B 46, 14578 (1992).

57. R.B. Campbell and C.A. Julien, J. Appl. Phys. 32, S346 (1961).

58. Z.A. Abdelnour, H.F. Mildrum, and K.J. Strnat, IEEE Trans. Magn. 17, 2651 (1981).

59. Q. Zeng, I. Baker, J.B. Cui, and Z.C. Yan, J. Magn. Magn. Mater. 308, 214 (2007).

60. C. Muller, H.H. Stadelmaier, B. Reinsch, and G. Petzow, Z. Metallkd. 88, 620 (1997).

61. L. Pareti, F. Bolzoni, F. Leccabue, and A.E. Ermakov, J. Appl. Phys. 59, 3824 (1986).

62. X.Z. Li, P. Kharel, V.R. Shah, and D.J. Sellmyer, Philos. Mag. 91, 3406 (2011).

63. P. Kharel, R. Skomski, and D.J. Sellmyer, J. Appl. Phys. 109, 07B709 (2011). doi:10.1063/1.3537952.

64. J.B. Yang, W.B. Yelon, W.J. James, Q. Cai, M. Kornecki, S. Roy, N. Ali, and P. l'Heritier, J. Phys.-Condens. Matter 14, 6509 (2002).

65. Y. Du, J. Wang, J.R. Zhao, J.C. Schuster, F. Weitzer, R Schmid-Fetzer, M. Ohno, H.H. Xu, Z.K. Liu, S.L. Shang, and W.W. Zhang, Int. J. Mater. Res. 98, 855 (2007).

66. J.F. Herbst, Rev. Mod. Phys. 63, 819 (1991).

67. C.D. Fuerst and J.F. Herbst, J. Appl. Phys. 69, 7727 (1991).

68. R.J. Parker, Advances in Permanent Magnetism (New York: Wiley-Interscience, 1990).

69. R. Skomski and J.M.D. Coey, Phys. Rev. B 48, 15812 (1993).

70. B. Balamurugan, D.J. Sellmyer, G.C. Hadjipanayisc, and R. Skomskia, Scripta Mater. 67 (2012). doi:10.1016/j.scripta mat.2012.03.034

71. R. Skomski, J. Phys.-Condens. Matter 15, R841 (2003).

72. Oak Ridge National Laboratory, Evaluation of the 2010 Toyota Prius Hybrid Synergy Drive System, ORNL/TM2010/253 (Oak Ridge, TN: Oak Ridge National Laboratory, 2011), http://info.ornl.gov/sites/publications/files/Pub26762. pdf. 